\title{
La conducta prosocial y su relación con los estilos parentales en adolescentes: educación pública y privada
}

Marcela Patricia del Toro Valencia ${ }^{1}$

\section{Introducción}

La conducta prosocial es entendida como todo acto realizado para ayudar o beneficiar a otro(s); ésta debe ser fomentada en adolescentes y jóvenes por ser un antídoto contra la conducta antisocial y la violencia. La promoción de este tipo de comportamiento de ayuda, asistencia, cooperación, solidaridad, entre otras, favorece conductas adaptativas y un adecuado ajuste social en este sector de la población, por lo que este tipo de conductas puede considerarse como una fortaleza para los adolescentes, en virtud de que juega un papel central en el bienestar psíquico y físico de los adolescentes (Martínez, Inglés, Piqueros y Oblitas, 2010).

En palabras de Holmgren, Eisenberg y Fabes (1998, en Sánchez-Queija, Oliva y Parra, 2006), el término de conducta prosocial hace referencia a los comportamientos llevados a cabo de una manera voluntaria para ayudar o beneficiar a otros, como serían: compartir, brindar apoyo y comprensión.

1 Universidad Michoacana de San Nicolás de Hidalgo. 
En este orden de ideas, Eisenberg (1986, en Fuentes, López, Etxebarria, Ledezma, Ortiz y Apodaca, 1993), describe la prosocialidad como aquel comportamiento voluntario que busca beneficiar a otras personas. También para Moñivas (1996), cualquier comportamiento que beneficia a otros o que conlleva consecuencias sociales positivas recibe el nombre de conducta prosocial.

Abocarse en el estudio y desarrollo de conductas prosociales en adolescentes y jóvenes permite hacer patente en ellos no sólo los atributos positivos que poseen todos los seres humanos sino también las conductas promotoras de la salud mental. Los adolescentes y los jóvenes son el sector de la formación y el cambio, su ente es de vital importancia para la célula social de actitud prosocial. En este orden de ideas, Vázquez, Hevás y Ho (2006, en Padrós, Martínez, Gutiérrez y Medina, 2010), afirman que para lograr una mejor comprensión del funcionamiento humano es necesario ir más allá de los aspectos negativos del individuo y considerar sus aspectos positivos. Así, desde hace más de una década, la psicología positiva, subsdiciplina de la psicología, ha centrado su interés en este tópico.

La psicología positiva busca el desarrollo de la persona a través de sus fortalezas o virtudes (Barragán, 2012). Seligman y Csikszentmihalyi (2001, en Castro, 2010), proponen para el siglo XXI una psicología que no sólo se dedique al estudio de reparar el daño psicológico sino también a investigar cómo se potencian las cualidades positivas que poseen todos los seres humanos.

Con fundamento en la psicología positiva es posible manifestar que cuando se llevan a cabo acciones encaminadas a ayudar y a beneficiar a otros, cuando se es solidario y se está dispuesto a cooperar con los más necesitados, destacan en el individuo rasgos positivos y emociones positivas, que son dos de los pilares básicos de la psicología positiva planteados por Castro (2010). Por tanto, promover comportamientos prosociales es dirigir la mirada hacia el estudio del funcionamiento mental óptimo (Seligman y Csikszentmihalyi, 2000).

Ahora bien, no podemos dejar de reconocer que la adopción de comportamientos positivos se gesta desde la familia; 
ésta constituye el contexto idóneo para el desarrollo de la conducta prosocial en adolescentes y jóvenes e influye de manera decisiva en la identidad y en la personalidad. El conjunto de relaciones que se establecen entre los miembros de la familia que comparten el mismo espacio y que constituyen el ambiente familiar determinan valores, actitudes y conductas que el niño va asimilando desde que nace.

Este ambiente familiar tiene funciones educativas y afectivas muy importantes. Como parte de éste, los estilos parentales de los padres tienen una gran influencia en el comportamiento de sus hijos, tanto en conductas agresivas y violentas en las que subyace hacer daño a otro(s), como en su opuesto, que son las conductas de índole prosocial que remiten a hacer el bien. De acuerdo con Garaigordobil (2005), una de las variables más importantes en la célula de la familia para el desarrollo de la conducta prosocial en niños y en adolescentes es el estilo parental, tanto del padre como de la madre.

Los estilos parentales son descritos como procesos a través de los cuales los padres intentan socializar a los hijos a través de prácticas emocionales y conductuales (Baumrind, 1966, 1971, 1980, 1993, 1991, en Palacios y Andrade, 2006).

En este orden de ideas, Darling y Steinberg (1993, en Palacios y Andrade, 2007), conciben el estilo parental como el conjunto de conductas que son comunicadas hacia el niño y que causan un clima emocional en el cual la conducta parental se expresa. Para los autores mencionados, existen evidencias empíricas que muestran que esas conductas tienen un efecto directo en la vida de los hijos.

Maccoby y Martin (1983, en Palacios y Andrade, 2007) aplican el concepto de estilo parental a las distintas cualidades de los padres, las cuales tienden a ser relativamente invariables a lo largo del tiempo y de las situaciones.

Con fundamento en lo antes expuesto se concluye que el estilo parental es la construcción psicológica que representa las estrategias estándar que los padres utilizan en la crianza del niño. El padre y la madre, por su historia, costumbres, tradiciones, personalidad y educación, entre otros aspectos, pueden 
utilizar diferentes estrategias que derivan en la conformación de cuatro estilos parentales, que son: el autoritario, el democrático, el negligente y el inconsistente.

Dependiendo de la manera en la que los adolescentes perciban el estilo parental de papá y mamá es el impacto que se tendrá en la crianza de los hijos y en su comportamiento. Uno de los aspectos involucrados en esta crianza es el desarrollo de conductas, tema de interés para la conducta prosocial. A partir de lo anterior, surge el objetivo del presente estudio, el cual es analizar si existe relación entre la conducta prosocial y los estilos parentales en adolescentes de escuelas públicas y privadas y determinar diferencias por variables contextuales.

\section{Método}

La investigación se basó en un enfoque cuantitativo con diseño no experimental, de alcance descriptivo-correlacional.

\section{Participantes}

La población total de estudio estuvo conformada por 175 estudiantes del nivel medio superior, de los cuales $52.3 \%$ fueron del sexo masculino y $47.7 \%$ del sexo femenino. La edad de los sujetos osciló entre 15 y 20 años con una media de 16; 59.1\% cursaba el segundo semestre del bachillerato y $40.9 \%$ el cuarto; $49.7 \%$ de los participantes asistía a una escuela pública y $50.3 \%$ a una privada, ambas ubicadas en la ciudad de Morelia, Michoacán.

\section{Instrumentos}

Para el logro del objetivo de este estudio se aplicó un cuestionario que fue diseñado ex profeso para obtener la información sociodemográfica. Para evaluar la conducta prosocial se utilizó la Escala de Habilidades Prosociales para Adolescentes (EAP-A), de Suárez y Morales (2011) que consta de 20 reactivos 
con cuatro opciones de respuesta; evalúa cuatro factores de la conducta prosocial: Toma de Perspectiva (TDP), Solidaridad y Respuesta de Ayuda (SYRA), Altruismo (ALT) y Asistencia (AST); su índice de confiabilidad es de $\alpha=.859$ y la varianza total explicada es de $47.15 \%$.

Los estilos parentales se midieron con la Escala de Estilos Parentales, de Palacios y Andrade (2006), que incluye prácticas parentales desde la percepción del adolescente mexicano y posibilita una tipología de estilos parentales: el autoritario, el democrático, el negligente y el inconsistente. El instrumento está conformado por una escala de 76 reactivos para papá y otra para mamá, con cuatro opciones de respuesta.

\section{Procedimiento}

La investigación se llevó a cabo en dos preparatorias de la ciudad de Morelia, Michoacán, una pública y otra privada, en donde se solicitó la autorización de las autoridades y la participación de los estudiantes.

Para el análisis de los datos se utilizó el programa sPss 17.0, obteniéndose la estadística descriptiva, y las pruebas estadísticas t de Student, Anova y Coeficiente de correlación de Pearson.

\section{Resultados}

A fin de determinar la existencia de asociación entre las variables de estudio, primeramente se obtuvo una descripción de las mismas, destacando que en cuanto a los factores de la conducta prosocial, en particular de toma de perspectiva, predominaron los niveles bajos, al igual que altruismo. En solidaridad arrojaron niveles medio bajo; y en asistencia llama la atención que predominaron de igual manera los niveles alto y bajo.

En relación con la percepción del estilo parental del padre predominó el estilo democrático, seguido del negligente. En cuanto a la forma en la que los adolescentes percibieron el estilo 
parental de la madre predominaron los estilos negligente y democrático.

En torno al objetivo del presente estudio no se encontró relación entre el estilo parental del padre y la conducta prosocial. Mientras que el estilo parental de la madre, el democrático, se asoció con solidaridad $(r=.372 ; p=.002)$ y altruismo $(r=.449 ; p=.000)$.

En cuanto al análisis comparativo, en relación con la conducta prosocial, se encontraron diferencias por escolaridad de la madre en altruismo. Los adolescentes cuyas madres cuentan con estudios de preparatoria se describen a sí mismos más altruistas que aquellos cuyas madres tienen estudios de primaria.

También se observaron diferencias por ocupación del padre en solidaridad y altruismo, resaltando en ambos casos que los adolescentes con padres profesionistas se describen a sí mismos más solidarios y altruistas que aquellos hijos de padres no profesionistas.

Además, se encontraron diferencias significativas en el estilo parental materno por escuela de procedencia $(t=-.270$; $p=.042$ ); los estudiantes de escuela privada perciben más en las madres el estilo parental inconsistente, mientras que en los estudiantes de la escuela pública predomina la percepción del estilo autoritario.

\section{Discusión}

Los adolescentes de la muestra mostraron tendencia hacia los niveles bajos en los factores de la conducta prosocial, fundamentalmente en solidaridad, altruismo y toma de perspectiva. Estos resultados indican que la mayoría de los adolescentes se describen con una capacidad baja para ponerse en el lugar del otro, comprender sus pensamientos, sentimientos y conductas, asistir y ayudar a otros, cooperar y ser solidarios, es decir, poco prosociales; sin embargo, en relación con el factor de asistencia llamó la atención la tendencia tanto a los niveles alto y bajo. Esto significa que una parte importante de los adolescentes de la muestra se concibieron como personas que ayudan a otros 
en situaciones específicas, mientras que un porcentaje similar percibió lo contrario.

Resultados de diversos estudios muestran que los adolescentes se autoperciben y se consideran más inadaptados que los niños o los adultos (Siverio y García, 2007, en Martínez, Inglés, Piqueras y Oblitas, 2010). Lo anterior se contradice con lo reportado por Mestré, Samper, Tur, Cortés y Nacher (2006), quienes encontraron que los adolescentes consideran que actúan prosocialmente y esta consideración se mantiene estable a lo largo de la etapa adolescente.

En relación con los estilos parentales, los resultados muestran que se reconoce a la madre como fuente de apoyo en el fomento de conductas prosociales en los adolescentes. Éstos caracterizaron a una madre con un estilo parental democrático que se identifica por brindar apoyo y afecto, a la vez que establece límites claros, resultados que coinciden con lo señalado por Mestre et al. (2007, en Cuervo, 2009), quienes señalan que cuando los adolescentes perciben que en la familia existe la convivencia definida por el apoyo emocional, principalmente brindado por parte de la madre, y la evaluación positiva del hijo, a la vez que existe coherencia en la aplicación de normas, que son características del estilo democrático, se alcanza un poder predictor de la conducta prosocial.

Con lo anterior resalta el papel de la madre en la adopción de comportamientos orientados a ayudar a otros en los hijos, y ello es clave, ya que por lo regular es la madre quien está al cuidado de los hijos, quien mantiene una comunicación más abierta y quien provee un modelo basado en conductas mayormente empáticas. Lo anterior ha sido corroborado por Retuerto (2004) al señalar que las mujeres alcanzan puntuaciones más altas que los varones en el afecto empático.

Los resultados también arrojan diferencias en la prosocialidad en función de la escolaridad de la madre, datos corroborados por Quintana, Montgomery y Malaver (2009), quienes afirman que la madre con experiencia escolar dota a los hijos de recursos para adaptarse a demandas internas y ambientales, y desarrollar conductas orientadas hacia los demás. 
De igual forma se registran diferencias por ocupación del padre en la conducta prosocial. Los resultados del estudio sugieren que es necesario promover comportamientos prosociales en niños, adolescentes y jóvenes para incidir en conductas adaptativas en ellos, por un lado, y, por otro, la sociedad se beneficiaría al estar integrada por individuos con conductas altamente prosociales.

\section{Referencias}

Barragán, A.R. (2012). Psicología positiva y humanismo: premisas básicas y coincidencias en los conceptos. Revista Electrónica de Psicología Iztacala.

Castro, A. (2010). Fundamentos de Psicología Positiva. Argentina: Paidós.

Cuervo, A. (2009). Pautas de crianza y desarrollo socioafectivo en la infancia. Diversitas: Perspectivas en Psicología, 6(1), 111-121.

Fuentes, M., López, F., Etxebarria, I., Ledezma, A., Ortiz, M. y Apodaca, P. (1993). Empatía, role-taking, y concepto de ser humano como factores asociados a la conducta prosocial/altruista. Infancia y Aprendizaje, 6, 55-78. Recuperado de http://www.ehu.es/pbwetbii/itziweb/PDFS\%20WEB/ Empat\%EDa,\%20role\%20taking.PDF.

Garaigordobil, M. (2005). Diseño y evaluación de un programa de intervención socioemocional para promover la conducta prosocial y prevenir la violencia. España: Ministerio de Salud.

Martínez, A., Inglés, C., Piqueras, J.A. y Oblitas, L.A. (2010). Papel de la conducta prosocial y de las relaciones sociales en el bienestar psíquico y físico del adolescente. Avances en Psicología Latinoamericana, 28(1), 74-84.

Mestre, M. V., Samper, P., Tur, A. M., Cortés, M. T., y Nácher, M. J. (2006). Conducta prosocial y procesos psicológicos implicados: Un estudio longitudinal en la adolescencia. Revista Mexicana de Psicología, 23(2), 203-215. 
Moñivas, A. (1996). Conducta pro-social. Cuadernos de trabajo social, 9, 125-142. Recuperado de http://revistas.ucm.es/ index.php/CUTS/article/view/CUTS9696110125A.

Padrós, F., Martínez, M., Gutiérrez, C. y Medina, M. (2010). La psicología positiva. Una joven disciplina científica que tiene como objeto de estudio un viejo tema, la felicidad, Uaricha, 14, 30-40.

Palacios, J. y Andrade, P. (2006). Escala de estilos parentales en adolescentes mexicanos. Revista de Psicología Social y Personalidad, 21(1), 50-52.

Quintana, A., Montgomery, W. y Malaver, C. (2009). Modos de afrontamiento y conducta resiliente en adolescentes espectadores de violencia entre pares. Revista de Investigación en Psicología, 12(1), 153-171.

Retuerto, A. (2004). Diferencias en empatía en función de las variables género y edad. Apuntes de Psicología, 22(3), 323339. Recuperado de http://www.cop.es/delegaci/andocci/ files/contenidos/vol22_3_3.pdf.

Sánchez-Queija, I., Oliva, A. y Parra, Ä. (2006). Empatía y conducta prosocial durante la adolescencia. Revista de Psicología Social, 21(3), 259-271. Recuperado de http:// personal.us.es/oliva/prosocial.pdf.

Suárez, C. y Morales, M. (2011). Construcción y validación de una escala para evaluar habilidades prosociales en adolescentes. Tesis de licenciatura. Universidad Michoacana de San Nicolás de Hidalgo, México.

Seligman, M. y Csikszentmihalgy, M. (2000). Positive Psychology: An Introduction. American Psychologist, 55, 5-14. 
